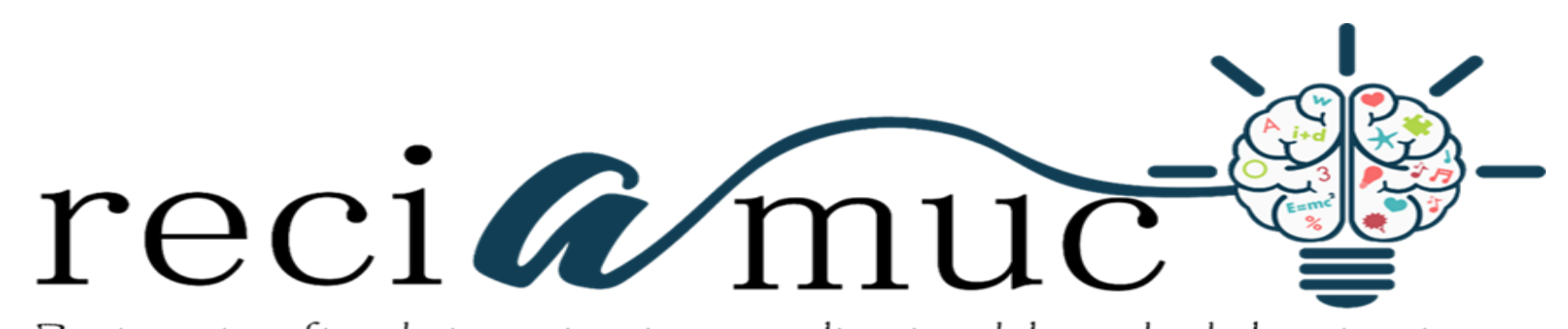

Revista cientifica de investigación actualización del mundo de las ciencias

\author{
Ana Gabriela Garzón Medina a ; Wilson Moisés Ortiz Contreras b; Karina Fernanda \\ Veliz Guadamud $^{\text {c. José María Cabezas Montes }}{ }^{\text {d }}$
}

Manejo postoperatorio de los anticoagulantes orales

Postoperative management of oral anticoagulant

Revista Científica de Investigación actualización del mundo de las Ciencias. Vol. 3 núm., 2, abril, ISSN: 2588-0748, 2018, pp. 459-487

DOI: $\underline{\text { 10.26820/reciamuc/3.(2).abril.2019.459-487 }}$

URL: http://reciamuc.com/index.php/RECIAMUC/article/view/349

Código UNESCO: 3205 Medicina Interna

Tipo de Investigación: Artículo de Revisión

(C) RECIAMUC; Editorial Saberes del Conocimiento, 2019

Recibido: 19/02/2019 Aceptado: 07/03/2019 Publicado: 01/04/2019

Correspondencia: anitagarzon27@gmail.com

a. Médico; Investigador Independiente; Guayaquil, Ecuador; anitagarzon27@gmail.com

b. Doctor en Medicina; Investigador Independiente; Guayaquil, Ecuador; dr.ortiz@outlook.es

c. Médico; Investigador Independiente; Guayaquil, Ecuador; karina_veliz@live.com

d. Médico; Magister en Emergencias Médicas; Especialista en Medicina Interna; Investigador Independiente; Guayaquil, Ecuador; anita_b_u@hotmail.com 


\section{Manejo postoperatorio de los anticoagulantes orales}

Vol. 3, núm. 2., (2019)

Ana Gabriela Garzón Medina; Wilson Moisés Ortiz Contreras; Karina Fernanda Veliz Guadamud; José María Cabezas Montes

\section{RESUMEN}

Toda actividad quirúrgica, sea de incisión, extirpación, manipulación o sutura de tejidos, debe ser realizada de forma eficiente en sus diferentes fases: preoperatoria, intraoperatoria y postoperatoria, siendo ésta última de interés para implantar acciones preventivas y correctivas que reduzcan posibles daños evitables. Se recomienda utilizar anticoagulantes como una alternativa viable para lograr efectividad durante la cicatrización, para reducir la formación de coágulos sanguíneos en las venas profundas de la pierna y evitar una trombosis venosa profunda o una embolia pulmonar. Por ello, los especialistas para equilibrar estos riesgos administran anticoagulantes orales los cuales impiden que el hígado utilice la vitamina $\mathrm{K}$, necesaria para elaborar algunos factores que intervienen en la coagulación; entre ellos: el acenocumarol y warfarina. Sin embargo, estos anticoagulantes orales actúan interfiriendo con la acción de la vitamina K, razón por la cual, es necesario mantener un control del paciente en cuanto a la alimentación, presencia de trastornos intestinales como diarrea o estreñimiento, pues, la absorción del anticoagulante por el tubo digestivo se verá afectada; de igual manera, ocurre ante la presencia de gastritis, vómitos o en el caso de aquellos pacientes tratados con plantas medicinales o homeopatía que pueden tener una intervención en la vitamina $\mathrm{K}$; de esta manera, el médico logra reducir los posibles efectos secundarios como complicaciones hemorrágicas o trombóticas; es allí, donde la incorporación del test del tiempo de protrombina se convierte en una herramienta esencial para mantener o reducir la dosis previamente medicada. Estos planteamientos, permiten dirigir la acción investigativa del presente artículo, cuyo objetivo es analizar el manejo postoperatorio de los anticoagulantes orales, para lo cual, se incorporaron técnicas que facilitaron su ubicación en el marco de las investigaciones documentales y construir un conjunto de apreciaciones generales como reporte de las interpretaciones realizadas a lo largo del hecho investigativo.

Palabras claves: Manejo postoperatorio; Anticoagulantes orales; Complicaciones hemorrágicas. 


\title{
Manejo postoperatorio de los anticoagulantes orales
}

Vol. 3, núm. 2., (2019)

Ana Gabriela Garzón Medina; Wilson Moisés Ortiz Contreras; Karina Fernanda Veliz

Guadamud; José María Cabezas Montes

\begin{abstract}
All surgical activity, incision, excision, manipulation or suture of fabrics, is should be carried out efficiently in different phases: preoperative, intraoperative, and postoperative, being the latter's interest to implement actions preventive and corrective to reduce avoidable damage. We recommend using anticoagulants as a viable alternative to achieve effectiveness during healing, to reduce the formation of blood clots in the deep veins of the leg and prevent deep vein thrombosis or pulmonary embolism. For this reason, specialists to balance these risks administered oral anticoagulants, which prevent the liver to use vitamin K, required to develop some factors involved in clotting; among them: the acenocoumarol and warfarin. However, these anticoagulants oral Act interfering with the action of the vitamin $\mathrm{K}$, reason by which, is necessary keep a control of the patient with regard to the power, presence of disorders intestinal as diarrhea or constipation, as, it anticoagulant absorption by the gastrointestinal tract is affected; Similarly, occurs in the presence of gastritis, vomiting or in the case of those patients treated with herbs or homeopathy that intervention can have on vitamin $\mathrm{K}$; in this way, the doctor manages to reduce the possible side effects such as thrombotic or hemorrhagic complications; It is there, where the incorporation of the prothrombin time test becomes an essential tool to maintain or reduce the doses of previously medicated. These approaches allow to direct the investigative action of this article, whose objective is to analyze the postoperative management of oral anticoagulants, which, incorporated techniques that facilitated its location within the framework of the documentary research and build a set of General appreciations such as interpretations made over the fact investigative report.
\end{abstract}

Key Words: Management postoperative; Anticoagulant oral; Hemorrhagic complications. 


\section{Manejo postoperatorio de los anticoagulantes orales}

Vol. 3, núm. 2., (2019)

Ana Gabriela Garzón Medina; Wilson Moisés Ortiz Contreras; Karina Fernanda Veliz Guadamud; José María Cabezas Montes

\section{Introducción.}

El organismo humano desde su punto de vista fisiológico se encuentra determinado por la presencia de un equilibrio entre las diferentes sustancias encargadas de lograr un proceso de coagulación. Es decir, la presencia de los anticoagulantes para mantener el estado fluido de la sangre. Cuando este equilibrio se rompe, lo que puede suceder por múltiples causas, surgen estados patológicos que pueden ser estados de hipocoagulabilidad (riego de hemorragias) o estados de hipercoagulabilidad (riesgo de trombosis).

El estado de hipercoagulabilidad, o su sinónimo trombofilia, puede definirse como cualquier alteración hereditaria o adquirida del balance entre factores procoagulantes y anticoagulantes que determine un aumento del riesgo de trombosis, es decir, de formación de coágulos en vasos sanguíneos (arterias, venas o ambos) que impiden el flujo de la sangre hacia los tejidos. El estado de hipercoagulabilidad puede suponer un importante problema de salud ya que puede ocasionar infartos o ictus. Por ello, para prevenir la formación de coágulos se emplean fármacos anticoagulantes. Estos favorecen la fluidez de la sangre en venas y arterias, pero no son efectivos cuando el coágulo ya se ha formado.

Los anticoagulantes orales clásicos actúan como Antagonistas de la Vitamina K (AVK). Impiden la transformación en el hígado de la vitamina $\mathrm{K}$ inactiva (epóxido) a vitamina $\mathrm{K}$ activa (hidroquinona), que es un cofactor necesario para el paso del ácido carboxiglutámico a ácido gammacarboxiglutámico en los residuos terminales de los factores II, VII, IX y X, y en las proteínas $\mathrm{C}$ y S. La ausencia de estos residuos ocasiona que estos factores sean inactivos por la incapacidad de ligar el calcio. 


\section{Manejo postoperatorio de los anticoagulantes orales}

Vol. 3, núm. 2., (2019)

Ana Gabriela Garzón Medina; Wilson Moisés Ortiz Contreras; Karina Fernanda Veliz

Guadamud; José María Cabezas Montes

En tal sentido, Reyes (2015) precisa que “se calcula que en España 13,2/1.000 habitantes reciben tratamiento con AVK, el cual se lleva a cabo con dicumarínicos, acenocumarol (aproximadamente el 90\% de los pacientes) o warfarina." (p.25). Según los datos referenciados permiten indicar que los AVK son fármacos de manejo complejo. Su margen terapéutico es estrecho y requieren monitorización periódica para conseguir mantener unos niveles razonables de seguridad y eficacia. Además, los AVK tienen múltiples interacciones con otros fármacos, con la ingesta dietética de vitamina $\mathrm{K}$ o alcohol, con enfermedades intercurrentes y otros factores. Aunque se han realizado avances significativos en su manejo, los AVK están implicados en gran número de ingresos hospitalarios, urgencias y complicaciones hemorrágicas.

En esta misma dirección, Ordovás (2018) destaca que actualmente se han creados nuevos anticoagulantes orales:

Para la prevención de la tromboembolia venosa (TEV) en adultos sometidos a cirugía de reemplazo de cadera o rodilla, siendo un nuevo inhibidor directo del factor Xa oral, rivaroxaban (Xarelto), para la prevención de formación de coágulos sanguíneos venosos en pacientes sometidos a cirugía electiva de sustitución total de cadera y rodilla. (p.18)

Según lo planteado, se puede decir que, para lograr una adecuada intervención favorable en pacientes sometidos a procesos quirúrgicos de rodilla y cadera, con el fin de evitar posibles situaciones vinculadas con hemorragias o trombosis venosa. Asimismo, el autor citado, precisa que los pacientes a los que se les realiza cirugía tienen un mayor riesgo de desarrollar coágulos sanguíneos en las venas. Estos coágulos pueden estar en las venas profundas de la pierna (trombosis venosa profunda (TVP) o llegar a los pulmones (embolia pulmonar EP). El 


\section{Manejo postoperatorio de los anticoagulantes orales}

Vol. 3, núm. 2., (2019)

Ana Gabriela Garzón Medina; Wilson Moisés Ortiz Contreras; Karina Fernanda Veliz Guadamud; José María Cabezas Montes

tromboembolismo venoso (TEV) es el término combinado para la TVP y la EP. La prevención de estos coágulos sanguíneos (profilaxis) después de la cirugía puede reducir el riesgo de coágulos venosos posoperatorios. Sin embargo, estos posibles efectos beneficiosos se deben equilibrar con los riesgos asociados de hemorragia. La duración óptima de la profilaxis después del reemplazo total de rodilla o cadera o la reparación de la fractura de cadera todavía es polémica.

En consecuencia, la recomendación médica en cuanto al uso de anticoagulantes orales, al paciente sometido a un proceso postoperatorio, recoge una significativa actuación, mediante ellos se logra disolver la sangre e impedir con ello la formación de coágulos que agranden y provoquen serios problemas de salud. Los mismos deben ser administrados por vía oral como una mecánica innovadora que ayuda al paciente a cumplir con el tratamiento desde su casa. Es decir, su incorporación puede administrarse a dosis fijas y no precisan sistemáticamente monitorización ni ajuste de dosis para asegurar su eficacia y seguridad.

Cada uno de los planteamientos citados, se convierten en elementos esenciales para la construcción del presente artículo, mediante las apreciaciones elaboradas, se estima el significado que tiene para la medicina moderna la consideración de los anticoagulantes orales en pacientes que cumplen un postoperatorio, dado que, los mismos debido a su innovación carecen de acciones dirigidas a manejar controles en cuanto de posibles cambios en la cicatrización, presencia de hemorragias entre otras. 


\section{Manejo postoperatorio de los anticoagulantes orales}

Vol. 3, núm. 2., (2019)

Ana Gabriela Garzón Medina; Wilson Moisés Ortiz Contreras; Karina Fernanda Veliz

Guadamud; José María Cabezas Montes

\section{Método.}

Para la realización de este trabajo se ha llevado a cabo una amplia revisión bibliográfica de diferentes bases de datos científicos, que fueron fundamentales para su elaboración, por lo tanto, se hace necesario considerar lo citado por Ramos (2017) el método científico "es una forma dinámica que lleva al investigador a encontrar diferentes alternativas para la construcción de nuevas ideas". (p.54). De allí, que cuando se hace el respectivo abordaje de un tema científico, es importante considerar el manejo de medios científicos ajustados a sus condiciones generales para llegar a plantear valoraciones significativas.

Dentro de este orden de ideas, se puede indicar que la utilización del método deductivo hizo posible desarrollar las apreciaciones en un contexto general, para luego obtener informaciones particulares que al ser compaginadas logran ofrecer importantes consideraciones, es así como, Ramos (ob.cit) indica que mediante el método deductivo "se puede organizar e interpretar el todo de una idea, representarla en escenarios separados para luego compaginar y formar un conjunto total”. (p.56). Este planteamiento, permite comprender que, mediante el manejo preciso de este método, el investigador en este caso particular, logra desglosar los criterios estimados en cuanto al manejo postoperatorio de los anticoagulantes orales de manera disgregada para finalmente elaborar nuevas consideraciones generales.

\section{Tipo de Investigación.}

El desarrollo metodológico del presente artículo, permite ubicarlo en el campo científico que le corresponde a las investigaciones documentales, pues, el trabajo estuvo centrado en la 


\section{Manejo postoperatorio de los anticoagulantes orales}

Vol. 3, núm. 2., (2019)

Ana Gabriela Garzón Medina; Wilson Moisés Ortiz Contreras; Karina Fernanda Veliz Guadamud; José María Cabezas Montes

recopilación de información bibliográfica, obtenida mediante la utilización de técnicas centradas en la lectura, selección de fuentes básicas y adecuación de los contenidos a tratar para luego elaborar el cuerpo teórico del mismo. En esta dirección, Gómez (2019) define la investigación documental "como un proceso científico, sistemático de indagación, recolección, organización, análisis e interpretación de la información”. (p.64).

De acuerdo con lo citado por Gómez, se puede decir que la presentación del artículo, tuvo como finalidad llegar a un análisis preciso y coherente en relación al tema vinculado con el manejo postoperatorio de los anticoagulantes orales, para ello, se hizo necesario adecuar las condiciones de los aportes referenciales, organizados en función a los contenidos previamente fijados.

\section{Fuentes Documentales.}

La investigación documental, como resultado a sus propósitos, requiere de la utilización de fuentes relacionadas con la indagación de información, es decir, el investigador debe seleccionar aquellas condiciones necesarias para el tema seleccionado, delimitándolo en un contexto particular y así elaborar el cuerpo informativo que guarda relación con el interés en estudio. Tal como lo apoya, Peña (2017) las fuentes documentales "permiten leer, valorar, recopilar, accionar y expresar ideas estimadas en otros criterios que aseguran el éxito del trabajo científico”. (p.26). Según el planteamiento, se precisa que, mediante la introducción de la lectura previa, se pudo organizar los aspectos generales del tema y al mismo tiempo, se logró su desarrollo en eventos particulares para mantener una correspondencia con el método seleccionado. 


\section{Manejo postoperatorio de los anticoagulantes orales}

Vol. 3, núm. 2., (2019)

Ana Gabriela Garzón Medina; Wilson Moisés Ortiz Contreras; Karina Fernanda Veliz

Guadamud; José María Cabezas Montes

\section{Técnicas de Recolección de Información.}

Durante la selección del proceso investigativo, se tuvo la necesidad de ubicar la información en contextos especiales, razón por la cual, se realizaron actividades previas vinculadas con la selección de los contenidos, su análisis interpretativo para así redactar los respectivos elementos que forman parte del artículo. Según Peña (ob.cit) “los trabajos documentales están caracterizados por la utilización de técnicas referidas a textos monográficos, esquemas lógicos, cuadros sinópticos, paráfrasis e interpretaciones críticas.”(p.32).

Este planteamiento, permite entender que la incorporación de cada una de las técnicas antes citadas, sirvieron para seleccionar los aspectos teóricos, estimarlos en una construcción interpretativa que hizo posible generar comparaciones entre los eventos, para luego incluir en ellos las respectivas valoraciones críticas, que se encargaron finalmente de preparar el contenido general del artículo a presentar.

\section{Resultados.}

Los aspectos desarrollados a continuación, responden a la necesidad de valorar los criterios referidos a los anticoagulantes orales, como posibles medicamentos que ayudan a reducir los efectos de formación de coágulos sanguíneos una vez que el paciente es sometido a una cirugía. Por ello, se desglosan en forma precisa y describa para garantizar una interpretación cónsona a las características del producto investigativo. 


\section{Manejo postoperatorio de los anticoagulantes orales}

Vol. 3, núm. 2., (2019)

Ana Gabriela Garzón Medina; Wilson Moisés Ortiz Contreras; Karina Fernanda Veliz Guadamud; José María Cabezas Montes

\section{Proceso de Coagulación Sanguíneo}

Con el fin de comprender desde una perspectiva más ajustada a las características temática, se hace necesario desarrollar apreciaciones vinculadas con la formación de la coagulación sanguínea, de esta manera, el lector podrá valorar la necesidad de incorporar los anticoagulantes orales durante un postoperatorio, Es decir, este proceso se encuentra representado por la presencia de varias etapas de formación de coágulos sanguíneos para detener la hemorragia se llama coagulación. Cuando toda la cascada de coagulación funciona correctamente, la sangre se acumula firme en el lugar de la herida y la hemorragia se detiene. Las personas con un trastorno hemorrágico, sin embargo, no forman coágulos fuertes de manera rápida o no los forman en absoluto.

De este modo, se comprende que la cascada de coagulación es un proceso químico complejo que utiliza hasta 10 proteínas diferentes (llamadas factores de coagulación sanguínea o factores de coagulación) que se encuentran en la plasma de la sangre. Dicho de manera simple, el proceso de coagulación hace que la sangre pase de su estado líquido al sólido en el lugar de la herida. Así es como funciona el proceso expuesto por, Márquez (2016)

Herida: Un desgarre pequeño en una pared del vaso sanguíneo (por ejemplo, de un corte en la piel o de una herida interna) provoca una hemorragia.

Constricción de los vasos sanguíneos: Para controlar la pérdida de sangre, el vaso sanguíneo se estrecha (a esto se le denomina constricción) para limitar el flujo de sangre a través del vaso sanguíneo. 


\section{Manejo postoperatorio de los anticoagulantes orales}

Vol. 3, núm. 2., (2019)

Ana Gabriela Garzón Medina; Wilson Moisés Ortiz Contreras; Karina Fernanda Veliz

Guadamud; José María Cabezas Montes

Tapón de plaquetas: En respuesta a la herida, se activan células diminutas en la sangre llamadas plaquetas. Las plaquetas se adhieren unas a otras en el lugar de la herida y forman un tapón. La proteína llamada factor von Willebrand (Von Willebrand Factor, VWF) ayuda a las plaquetas a permanecer adheridas entre sí y hacia la pared del vaso sanguíneo.

Coágulo de fibrina: Luego, las proteínas del factor de coagulación activan la producción de la fibrina, una sustancia fuerte y parecida a una cuerda que forma un coágulo de fibrina, una red con forma de malla que mantiene al tapón firme y estable. A lo largo de los siguientes días y semanas, el tapón se fortalece y luego se disuelve a medida que la pared lesionada del vaso sanguíneo sana.

Imagen $\mathbf{N}^{\circ} 1$ Formación del Coagulo Sanguíneo

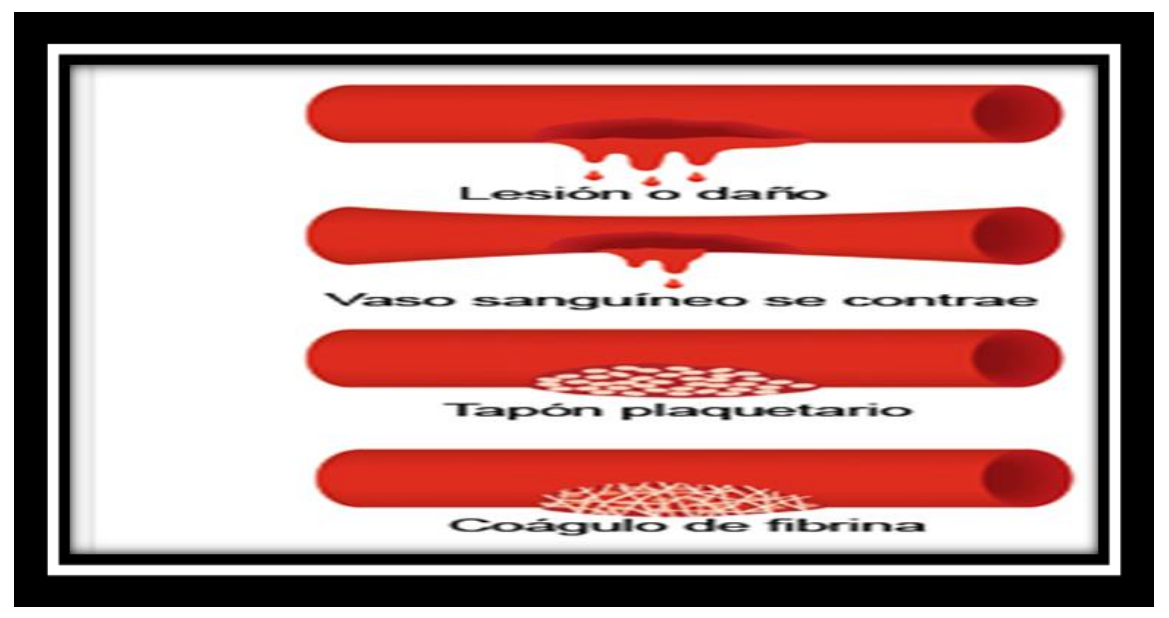

Fuente: Márquez (ob.cit)

Sin embargo, cuando una persona tiene un trastorno hemorrágico como la enfermedad de von Willebrand no tienen suficientes proteínas VWF o la proteína VWF no funciona correctamente. Al tener una hemorragia, no pueden formar un tapón de plaquetas. Además, el 


\section{Manejo postoperatorio de los anticoagulantes orales}

Vol. 3, núm. 2., (2019)

Ana Gabriela Garzón Medina; Wilson Moisés Ortiz Contreras; Karina Fernanda Veliz Guadamud; José María Cabezas Montes

VWF actúa como una proteína portadora para el factor VIII (FVIII), uno de los factores de coagulación en el plasma. El VWF ayuda a asegurar que haya suficiente FVIII en la sangre y que llegue a donde se necesita. Sin el VWF, el FVIII se deshará en el torrente sanguíneo y es posible que no llegue a ser suficiente para detener la hemorragia. Asimismo, cuando una persona tiene hemofilia, el vaso sanguíneo se estrecha y las plaquetas forman un tapón, pero falta o está dañada una de las proteínas del factor de coagulación que es esencial para una fibrina de coagulación firme, por lo que no se forma el coágulo o no es lo suficientemente fuerte como para detener la hemorragia. Esta es la razón por la que una persona con hemofilia sangra durante un período más largo.

Cabe destacar que, para controlar el sangrado, se originan diferentes factores de coagulación; es decir, cuando un vaso sanguíneo se lesiona, sus paredes se contraen para limitar el flujo de sangre al área dañada. Entonces, pequeñas células llamadas plaquetas se adhieren al sitio de la lesión y se distribuyen a lo largo de la superficie del vaso sanguíneo. Al mismo tiempo, pequeños sacos al interior de las plaquetas liberan señales químicas para atraer a otras células al área y hacer que se aglutinen a fin de formar lo que se conoce como tapón plaquetario.

En la superficie de estas plaquetas activadas muchos factores de coagulación diferentes trabajan juntos en una serie de reacciones químicas complejas (conocidas como cascada de la coagulación) para formar un coágulo de fibrina. El coágulo funciona como una red para detener el sangrado. Los factores de la coagulación circulan en la sangre sin estar activados. Cuando un vaso sanguíneo sufre una lesión se inicia la cascada de la coagulación y cada factor de la coagulación 


\section{Manejo postoperatorio de los anticoagulantes orales}

Vol. 3, núm. 2., (2019)

Ana Gabriela Garzón Medina; Wilson Moisés Ortiz Contreras; Karina Fernanda Veliz

Guadamud; José María Cabezas Montes

se activa en un orden específico para dar lugar a la formación del coágulo sanguíneo. Los factores de la coagulación se identifican con números romanos.

De este modo, se entiende la importancia que tiene para el ser humano, el proceso de coagulación sanguínea, dado que, la sangre circula por el sistema vascular, el cual está compuesto por el corazón y vasos sanguíneos. El corazón bombea la sangre a través de los vasos. Esta se compone de diversas células, las cuales son transportadas en un líquido llamado plasma. Las células rojas transportan oxígeno. Las células blancas forman parte del sistema inmunitario. Las plaquetas son vitales para la coagulación de la sangre. En estado natural, la sangre fluye sin producirse su coagulación. La pared del vaso está intacta, y no hay una mayor tendencia a la formación de coágulos. En este estado, anticoagulación y coagulación se hallan en equilibro.

Figura $\mathbf{N}^{\circ} 2$ Fisiología de la Hemostasis

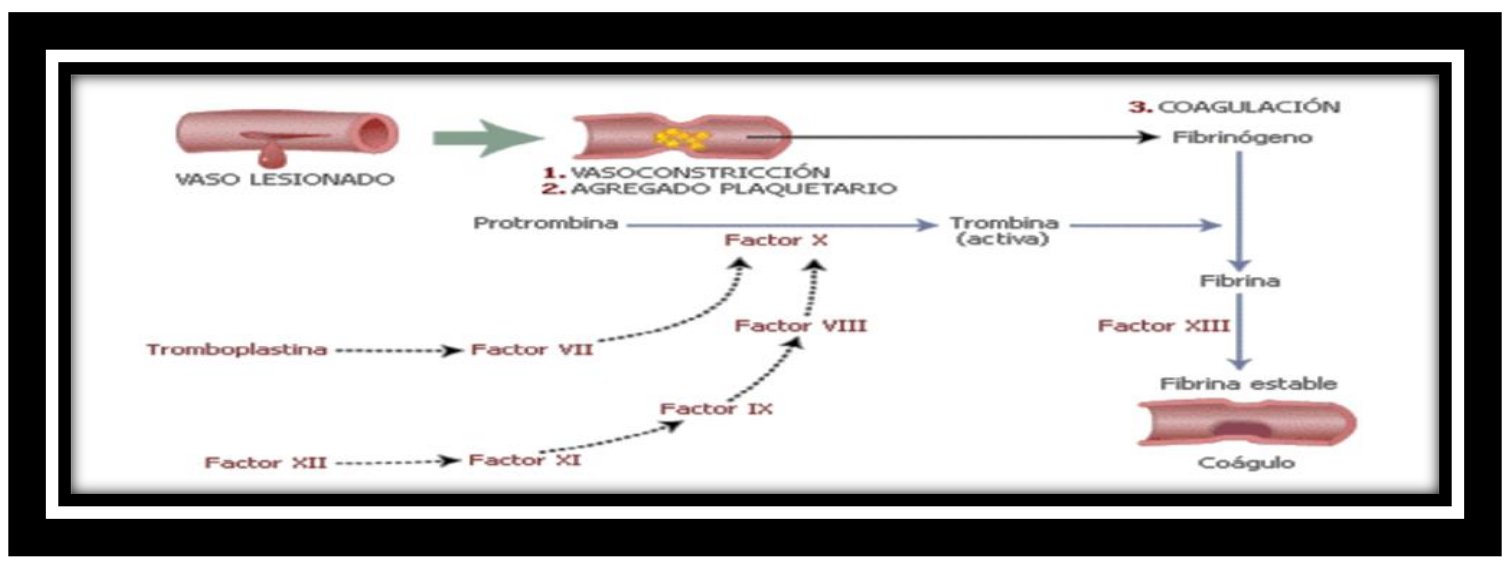

Fuente: Márquez (ob.cit)

La figura que antecede, permite evidenciar que la hemostasia es un proceso dinámico que permite al organismo detener el daño producido por una lesión a nivel vascular, mantener la sangre 


\section{Manejo postoperatorio de los anticoagulantes orales}

Vol. 3, núm. 2., (2019)

Ana Gabriela Garzón Medina; Wilson Moisés Ortiz Contreras; Karina Fernanda Veliz Guadamud; José María Cabezas Montes

en un estado fluido en condiciones fisiológicas y finalmente retirar el coágulo formado tras la reparación del daño vascular. En este proceso pueden distinguirse tres etapas: La hemostasia primaria: consiste en la adhesión, activación y agregación plaquetaria al endotelio vascular dañado con el fin de formar el coágulo hemostático primario o tapón plaquetario. La hemostasia secundaria o coagulación: activación de proteínas de la coagulación que acaban en la formación de un coágulo de fibrina. La fibrinólisis: consiste en la disolución del coágulo de fibrina una vez que se ha reparado el endotelio vascular.

Al respecto, Naranjo (2017), indica que "la formación de coágulos es esencial para la preservación de la vida; en caso de que se produzcan daños en un vaso", (p.15) Es decir, la lesión provoca una reacción en cadena que conduce a la formación de trombos. Una cantidad cada vez mayor de plaquetas se une al tejido dañado. El factor coagulante trombina, una proteína soluble que se encuentra en la sangre, juega un papel central en la formación de trombos. La trombina activa transforma en fibrina al fibrinógeno que se encuentra diluido en la sangre, formándose una malla estable las células sanguíneas quedan atrapadas en esta red, originándose así un coágulo que sella el vaso sanguíneo.

Asimismo, se puede indicar que los coágulos sanguíneos son masas que se presentan cuando la sangre se endurece pasando de líquida a sólida. Un coágulo sanguíneo que se forma dentro de una de las venas o las arterias se denomina trombo que se desprende y viaja desde un lugar en el cuerpo a otro se llama émbolo. Un trombo o émbolo puede bloquear parcial o totalmente el flujo de sangre en un vaso sanguíneo. Una obstrucción en una arteria puede impedir que el oxígeno llegue a los tejidos en esa área. Esto se denomina isquemia. Si la isquemia no se trata 


\section{Manejo postoperatorio de los anticoagulantes orales}

Vol. 3, núm. 2., (2019)

Ana Gabriela Garzón Medina; Wilson Moisés Ortiz Contreras; Karina Fernanda Veliz

Guadamud; José María Cabezas Montes

oportunamente, puede provocar daños en los tejidos o la muerte. Una obstrucción en una vena generalmente provocará acumulación de líquido e hinchazón.

Figura $\mathbf{N}^{\circ} 3$ Coágulo de Sangre o Trombo

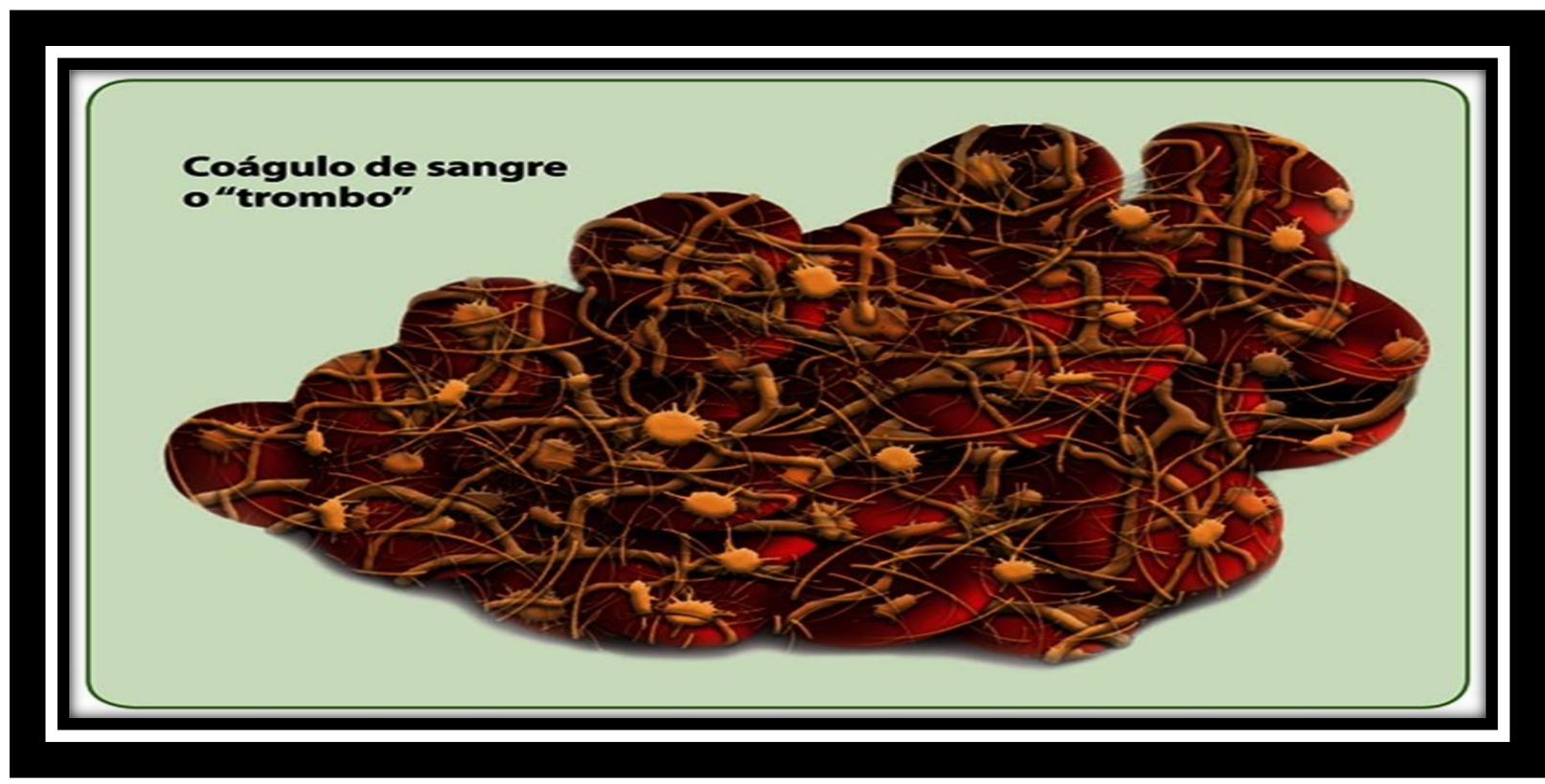

Fuente: Naranjo (ob.cit)

Es importante acotar que, un coágulo de sangre es una afección grave que requiere de tratamiento inmediato. Las personas con cáncer y las que reciben tratamiento para el cáncer tienen un mayor riesgo de formación de coágulos sanguíneos. El proceso normal, llamado coagulación, es un proceso complejo. En él participan células sanguíneas especializadas, denominadas plaquetas, y distintas proteínas de la sangre, denominadas factores de coagulación. Estas plaquetas y estos factores de coagulación se aglutinan para cicatrizar los vasos sanguíneos rotos y controlar el sangrado. Los factores de coagulación que promueven el sangrado y aquellos que promueven la coagulación deben estar equilibrados. 


\section{Manejo postoperatorio de los anticoagulantes orales}

Vol. 3, núm. 2., (2019)

Ana Gabriela Garzón Medina; Wilson Moisés Ortiz Contreras; Karina Fernanda Veliz Guadamud; José María Cabezas Montes

Sin embargo, al encontrar los trastornos de coagulación de la sangre se presentan cuando faltan o se dañan algunos factores de coagulación. Esto produce la formación de coágulos dentro del cuerpo que bloquean el flujo normal de la sangre y causan problemas graves. Es decir, los coágulos de sangre pueden producirse en diferentes partes del cuerpo y desplazarse a estas, por ejemplo: Las venas, lo cual se denomina trombosis venosa profunda. Los pulmones, lo cual se denomina embolia pulmonar. Una arteria (menos frecuente, pero también muy grave). Por ello, ante estas situaciones, se amerita un tratamiento inmediato. El tratamiento más frecuente consiste en administrar diluyentes de la sangre mediante inyección, ya sea debajo de la piel o en la vena. Cuando se considera que la sangre está lo suficientemente diluida, ya no hay riesgo de coagulación. En este momento, algunas personas pueden comenzar a tomar un anticoagulante en forma de comprimido que se traga.

A las personas que reciben anticoagulantes se las debe controlar regularmente para detectar cualquier aumento en el sangrado. Algunas personas no pueden recibir anticoagulantes porque tienen bajos niveles de plaquetas o un riesgo alto de sangrado. Para estas personas, se puede colocar un tipo especial de filtro en el cuerpo para evitar que un coágulo sanguíneo se desplace a los pulmones, lo que constituye una afección que puede ser muy peligrosa. Cada una de las consideraciones citadas, llevan a destacar la importancia clínica que tienen los anticoagulantes orales para pacientes sometidos a una práctica quirúrgica. 


\section{Manejo postoperatorio de los anticoagulantes orales}

Vol. 3, núm. 2., (2019)

Ana Gabriela Garzón Medina; Wilson Moisés Ortiz Contreras; Karina Fernanda Veliz

Guadamud; José María Cabezas Montes

Figura $\mathbf{N}^{\circ} 4$ Trombosis Venosa Pofunda y Edema Pulmonar

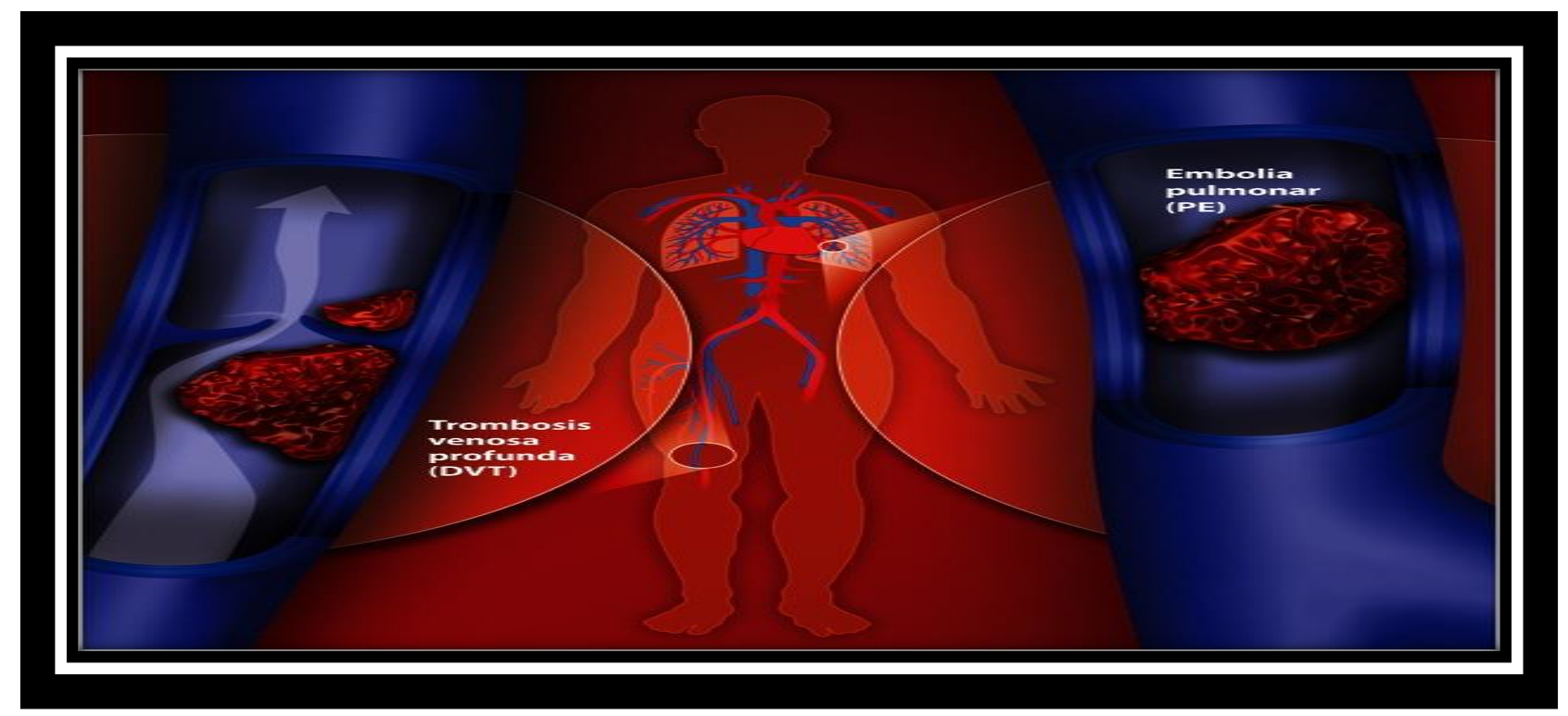

Fuente: Naranjo (ob.cit)

De este modo, la actitud ante cualquier complicación hemorrágica dependerá de la importancia y localización del sangrado, así como del nivel de anticoagulación. Hemorragias con riesgo vital inminente: hemorragias intracraneales, sangrado digestivo severo. Se derivarán al servicio de cirugía, donde se corregirá de inmediato el defecto coagulativo. Hemorragias importantes no peligrosas para la vida: hemorragias digestivas controlables, hematuria intensa, equimosis grandes espontáneas, pero en localizaciones no peligrosas, entre otros. También se derivan las cirugías para su corrección. Hemorragias de menor importancia: La epistaxis no severa, hemorragia subconjuntival, equimosis grandes espontáneas, pero en localizaciones no peligrosas.

Según la intensidad y localización, si se requiere, se reducirá o suspenderá la anticoagulación uno o dos días, continuado posteriormente con la dosis ambulatoria habitual, según el INR previo. Si se realiza supresión de anticoagulación más de un día habrá de considerar 


\section{Manejo postoperatorio de los anticoagulantes orales}

Vol. 3, núm. 2., (2019)

Ana Gabriela Garzón Medina; Wilson Moisés Ortiz Contreras; Karina Fernanda Veliz Guadamud; José María Cabezas Montes

la posibilidad de administrar heparina. En caso de prolongación excesiva del INR o si se considera necesario, se puede administrar vitamina $\mathrm{K}$ vía oral.

\section{Anticoagulantes Orales}

La terapia anticoagulante es ampliamente utilizada en la práctica clínica, como profilaxis en pacientes con riesgo de presentar fenómenos tromboembólicos o como tratamiento en aquellos que han presentado algún evento trombótico. Cada vez es más frecuente enfrentarse a pacientes en tratamiento anticoagulante crónico que serán intervenidos mediante procedimientos quirúrgicos, por lo que es importante y necesario conocer el manejo postoperatorio de los diferentes fármacos anticoagulantes, para disminuir los riesgos y complicaciones asociados a la suspensión o mantención de estos en dicho período. Para lograr este objetivo se debe evaluar y balancear el riesgo de sangrado versus el riesgo de eventos tromboembólicos, considerando la condición de cada paciente y el tipo de procedimiento quirúrgico que recibido.

Cabe destacar que, los nuevos anticoagulantes orales no requieren monitorización de rutina, recomendándose suspenderlos a las 24-96h previas al procedimiento quirúrgico, dependiendo del riesgo hemorrágico de cada cirugía y de la función renal. Existen múltiples alternativas de TAC, destacando dos grandes grupos: terapia anticoagulante oral (TACO), donde se encuentran los fármacos antagonistas de vitamina $\mathrm{K}$ (cumarínicos) y los nuevos anticoagulantes orales.

Al considerar este apartado, se puede decir que los mismos representan a un grupo de sustancias utilizados en tratamientos de mantención de distinta naturaleza química relacionada por su efecto biológico; asimismo, presentan una denominación genérica a la excelente 


\section{Manejo postoperatorio de los anticoagulantes orales}

Vol. 3, núm. 2., (2019)

Ana Gabriela Garzón Medina; Wilson Moisés Ortiz Contreras; Karina Fernanda Veliz

Guadamud; José María Cabezas Montes

biodisponibilidad que tienen cuando se administran por vía oral. Los más utilizados son la warfarina y el acenocumarol (Neo-sintrom), ambos derivados del dicumarol. Su mecanismo de acción, es inhibir el ciclo de interconversión de la vitamina $\mathrm{K}$ desde su forma oxidada a la reducida. Pues, la misma es el cofactor esencial para la síntesis hepática de las denominadas proteínas vitamina $\mathrm{K}$ dependientes. Sin embargo, se ha demostrado que su efecto anticoagulante se debe principalmente a la disminución de los niveles plasmáticos de protrombina funcional.

En consecuencia, la warfarina y acenocumarol (Neosintrom) son antagonistas de vitaminaK que alteran la coagulación impidiendo la síntesis de factores procoagulantes dependientes de dicha vitamina: II, VII, IX y X. La misma tiene una vida media de 36-42h y el acenocumarol, de 8-11h. Su efecto anticoagulante se mide con los exámenes tiempo de protrombina (TP). Estos fármacos se indican con esquemas de dosis individualizados para cada paciente, realizándose un control semanal del INR, el cual se considera en rango terapéutico con valores entre 2-3. Su utilización se hace a las 12-24h posteriores a procedimientos quirúrgicos con bajo riesgo de sangrado postoperatorio y asegurando una adecuada hemostasia postoperatoria. Una vez iniciado el tratamiento se demora entre 5-10 días en lograr un INR en rango terapéutico. Por tanto, en pacientes con alto riesgo tromboembólico se recomienda el uso de terapia puente postoperatoria asociada a warfarina, realizándose traslape entre ambos fármacos hasta lograr un INR en rango óptimo.

No obstante, la suspensión de dicho anticoagulante, puede ser aplicada en procedimientos quirúrgicos con bajo potencial de sangrado, como cirugía de cataratas, algunas intervenciones endoscópicas y procedimientos dermatológicos menores. La misma puede ser reiniciada. 


\section{Manejo postoperatorio de los anticoagulantes orales}

Vol. 3, núm. 2., (2019)

Ana Gabriela Garzón Medina; Wilson Moisés Ortiz Contreras; Karina Fernanda Veliz Guadamud; José María Cabezas Montes

Especialmente el acenocumarol, más conocido como sintrom, aunque también está disponible la warfarina (aldocumar). Medicamentos que actúan sobre la vitamina K, necesaria para que funcione todo el mecanismo de la coagulación. Se utilizan para la prevención a largo plazo de diversas complicaciones tromboembólicas. En cardiología su uso más frecuente es en la fibrilación auricular y en las valvulopatías (prótesis valvulares). Su intervención en la vía de la vitamina $\mathrm{K}$, presenta gran variabilidad de respuesta por lo que siempre es necesario un control estricto del nivel de anticoagulación.

Es importante destacar que, en la actualidad, existen cuatro nuevos anticoagulantes con desarrollo clínico más avanzado son dabigatrán, rivaroxabán, apixabán y edoxabán. Su farmacología y su farmacocinética. Al contrario que los anticoagulantes disponibles hasta el momento, estos fármacos inhiben sus dianas terapéuticas (la trombina o el factor Xa) directamente, en lugar de a través de un cofactor u otros mecanismos indirectos. Aunque su unión a la zona catalítica de la trombina o del factor Xa es reversible, actualmente no existen antídotos. Su inicio de acción es rápido, tanto como el de las heparinas subcutáneas. Hay otros fármacos en desarrollo, como el darexabán y el betrixabán (inhibidores del factor Xa y de la trombina).

De lo anterior, se desprenden que la utilización de estos nuevos anticoagulantes orales, incluyen los inhibidores directos de trombina y los inhibidores del factorX activado (Xa). Actualmente estos fármacos son ampliamente usados en clínica dado que no requieren monitorización rutinaria del efecto anticoagulante, presentan un efecto anticoagulante predecible y una eficacia similar a los anticoagulantes clásicos. Solo requieren monitorización en situaciones clínicas determinadas, como hemorragia aguda, sospecha de sobredosis o cirugía permiten generar 


\section{Manejo postoperatorio de los anticoagulantes orales}

Vol. 3, núm. 2., (2019)

Ana Gabriela Garzón Medina; Wilson Moisés Ortiz Contreras; Karina Fernanda Veliz

Guadamud; José María Cabezas Montes

cambios importantes en los pacientes que se encuentran en un postoperatorio; es así como, Mateo (2017) describe de manera precisa la actuación de los nuevos anticoagulantes orales, como antagonistas de la vitamina $\mathrm{K}$, entre ellos se citan los siguientes:

Rivaroxabán: Es un inhibidor potente y selectivo del factor Xa. Se une al centro activo del factor Xa y lo inhibe de manera reversible y competitiva. Inhibe el factor Xa libre y el Xa unido en el complejo protrombinasa. Se absorbe vía oral y su biodisponibilidad es superior al 80\%. La comida no interfiere en su absorción. El pico plasmático se consigue a las $3 \mathrm{~h}$ y la semivida es de 5-9h en adultos jóvenes y 11-13 h en ancianos. Un tercio se excreta vía renal sin metabolizar, y el resto de forma inactiva vía renal y en heces en partes iguales. Como otros inhibidores directos del factor Xa, el rivaroxabán prolonga el tiempo de protrombina y reduce el tiempo de tromboplastina parcial activado (TTPA). El mejor test para monitorizar su concentración en plasma es la dosificación de unidades de inhibición del factor Xa (anti-Xa). Carece de antídoto, pero hay datos preclínicos de que la administración de concentrado de factores del complejo protrombínico puede ser de utilidad para corregir las alteraciones biológicas en la hemostasia. No obstante, esto puede no reflejar su eficacia en el sangrado producido por el rivaroxabán.

Este medicamento, es el primer anticoagulante inhibidor directo de la trombina disponible por vía oral. La absorción del profármaco dabigatran etexilato y su conversión a dabigatran es rápida (concentraciones máximas de 4-6 h tras cirugía y $2 \mathrm{~h}$ posteriormente) y, pese a la baja biodisponibilidad oral, presenta escasa variabilidad entre individuos. Inhibe específica y reversiblemente la trombina, la enzima llave de la cascada de la coagulación. 


\section{Manejo postoperatorio de los anticoagulantes orales}

Vol. 3, núm. 2., (2019)

Ana Gabriela Garzón Medina; Wilson Moisés Ortiz Contreras; Karina Fernanda Veliz Guadamud; José María Cabezas Montes

De igual manera, se precisa que, mediante su utilización en diferentes grupos de pacientes bajo la presentación de 2,5mg/12h, otro con $5 \mathrm{mg} / 12 \mathrm{~h}$ y otro con placebo. Se observó que el rivaroxabán, comparado con placebo, reducía de manera significativa el objetivo de eficacia el 8,9 y el 10,7\%. Además, la dosis de $2,5 \mathrm{mg} / 12 \mathrm{~h}$ redujo la mortalidad cardiovascular (el 2,7 contra el $4,1 \% ; p=0,002)$ y la mortalidad por cualquier causa (el 2,9 contra el 4,5\%; $p=0,002)$. Mientras que con la dosis de $5 \mathrm{mg} / 12 \mathrm{~h}$ del medicamento aumentó el sangrado mayor (el 2,1 frente al 0,6\%; $\mathrm{p}<0,001)$ y la hemorragia intracraneal (el 0,6 frente al $0,2 \%$; $=0,009)$ sin incremento de sangrado mortal. En cambio, la dosis baja de rivaroxabán produjo menos hemorragias mortales que la más alta (el 0,1 frente al $0,4 \% ; p=0,04)$.

Apixabán: Es un inhibidor selectivo y reversible del centro activo del factor Xa. Al igual que el rivaroxabán, inhibe el factor Xa libre y el que está unido en el complejo protrombinasa. Se absorbe vía oral y su biodisponibilidad es superior al 50\%. El pico plasmático se consigue a las $3 \mathrm{~h}$ y su semivida puede oscilar entre 8 y $15 \mathrm{~h}$. Aproximadamente el $25 \%$ se excreta vía renal, mientras el resto aparece en las heces. La alteración de las pruebas de hemostasia es similar a la que produce el rivaroxabán. Carece de antídoto y es posible que la administración de concentrado de factores del complejo protrombínico sea de utilidad.

Dabigatrán etexilato: Se transforma por las esterasas en dabigatrán, que es su metabolito activo. Es un inhibidor directo de la trombina. La biodisponibilidad vía oral es baja, del 6\%. El pico plasmático se consigue en $2 \mathrm{~h}$ y la semivida es de $8 \mathrm{~h}$ tras una dosis única y de $12-17 \mathrm{~h}$ tras múltiples dosis. El 80\% se elimina vía renal sin metabolizar. El dabigatrán prolonga el TTPA y tiene un efecto mínimo en el tiempo de protrombina. Prolonga el tiempo de trombina de una 


\section{Manejo postoperatorio de los anticoagulantes orales}

Vol. 3, núm. 2., (2019)

Ana Gabriela Garzón Medina; Wilson Moisés Ortiz Contreras; Karina Fernanda Veliz

Guadamud; José María Cabezas Montes

manera dependiente de la dosis. Aunque esta es una prueba habitual en los laboratorios de hemostasia y es muy sensible a fármacos inhibidores de la trombina, no es útil para monitorizar su efecto por ser demasiado sensible. Existe una variación del tiempo de trombina, el tiempo de trombina diluido con plasma, que tiene una excelente correlación con la concentración plasmática del dabigatrán. El dabigatrán también prolonga el tiempo de ecarina de manera dependiente de la dosis. El tiempo de ecarina y el tiempo de trombina diluido con plasma son las pruebas más recomendables para evaluar las concentraciones de dabigatrán.

Cabe agregar que el medicamento, por ser un inhibidor directo de la trombina. Tras su administración oral se absorbe en el tracto gastrointestinal, alcanza su máxima concentración en plasma entre 30 minutos y 2 horas después y tiene una vida media de 12-14 horas. Dabigatrán inhibe competitivamente la trombina humana, de manera rápida y altamente selectiva, aunque reversible. Aproximadamente el $80 \%$ se excreta, sin cambios, por vía renal y el $20 \%$ restante se elimina por la bilis. Dado que el citocromo P450 no está involucrado en el metabolismo del fármaco tiene pocas interacciones. Es más, no es preciso limitar la ingesta de alimentos que contengan vitamina $\mathrm{K}$, son estables, lo que permite utilizarla a dosis fija, sin necesidad de monitorizar la coagulación.

De los planteamientos, citados anteriormente se puede indicar que la utilización de estos nuevos anticoagulantes orales, busca llevar a cabo la prevención y tratamiento de la tromboembolia venosa, la prevención del ictus y embolia de origen cardiaco en pacientes con fibrilación auricular (FA) no valvular y en prevención secundaria después de un síndrome coronario agudo. Para la profilaxis de la tromboembolia venosa tras cirugía ortopédica, están 


\section{Manejo postoperatorio de los anticoagulantes orales}

Vol. 3, núm. 2., (2019)

Ana Gabriela Garzón Medina; Wilson Moisés Ortiz Contreras; Karina Fernanda Veliz Guadamud; José María Cabezas Montes

aprobados el rivaroxabán, apixabán y dabigatrán, porque en diversos ensayos clínicos han demostrado eficacia y seguridad similares a las de la enoxaparina, y en algunos aspectos son superiores. El edoxabán está disponible en Japón. El rivaroxabán está aprobado como tratamiento inicial de la trombosis venosa profunda, de la embolia pulmonar y también en la prevención de recurrencias a largo plazo (estudios Einstein-DVT, Einstein-PE y Einstein-EXT). El dabigatrán ha mostrado eficacia y seguridad similares a las de la warfarina en el tratamiento de la tromboembolia venosa.

Cuadro $N^{\circ} 1$ Concentración de los Nuevos Anticoagulantes Orales

\begin{tabular}{|l|c|c|c|}
\hline \multicolumn{1}{|c|}{ Farmacocinética } & Dabigatrán & Rivaroxabán & Apixabán \\
\hline Pico de concentración plasmática (horas) & $1-25-2$ & $2-4$ & $2-3$ \\
\hline Vida media (rango en horas) & $12-14$ & $9-12$ & $8-15$ \\
\hline Eliminación Renal & SI & NO & NO \\
\hline
\end{tabular}

Fuente: Elaboración Propia (2019)

Sin embargo, estos nuevos anticoagulantes orales, como antagonistas de la vitamina $\mathrm{K}(\mathrm{AVK})$ han sido los únicos anticoagulantes orales disponibles en el último medio siglo. A pesar de suponer un importante avance terapéutico, también conllevan múltiples limitaciones como son el estrecho margen terapéutico, las interacciones con alimentos u otros fármacos, la necesidad de monitorización o los subsecuentes ajustes de dosis. 


\section{Manejo postoperatorio de los anticoagulantes orales}

Vol. 3, núm. 2., (2019)

Ana Gabriela Garzón Medina; Wilson Moisés Ortiz Contreras; Karina Fernanda Veliz Guadamud; José María Cabezas Montes

Cuadro $\mathbf{N}^{\circ} 2$ Actuación de la Warfarina y los Nuevos Anticoagulantes Orales.

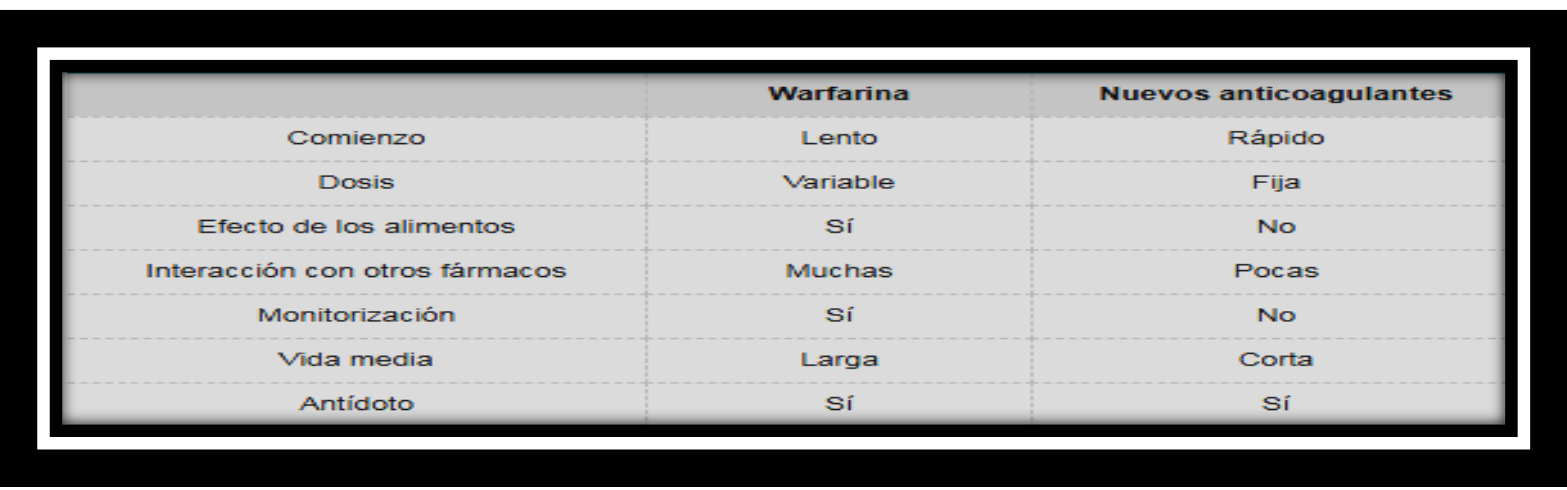

Fuente: Mateo (ob.cit)

Los elementos aportados en el cuadro 2, llevan a precisar que la actuación de los nuevos anticoagulantes orales, tienen una actuación más rápida en algunos rangos. En otras palabras, la alta prevalencia de fenómenos tromboembólicos y eventos cardiovasculares en la población ha masificado el uso de fármacos anticoagulantes, por lo que el equipo quirúrgico se verá enfrentado cada vez con mayor frecuencia a pacientes que serán intervenidos mediante algún tipo de cirugía. Existen diversas opciones de TAC, como también procedimientos quirúrgicos de toda índole y diferente riesgo de sangrado, por lo que las decisiones en relación con el manejo postoperatorio de estos fármacos deben ser adaptadas a cada paciente en particular y tomar en cuenta el riesgo beneficio de suspender o no la TAC durante el período postoperatorio.

En razón de lo anterior, se puede resaltar que los anticoagulantes orales, que son derivados dicumarínicos, actúan inhibiendo varios de los pasos para la transformación de la vitamina K activa en el hígado. La vitamina K es esencial para la síntesis de los factores de la coagulación II, VII, IX y X, así como las proteínas anticoagulantes C y S. La warfarina, es el medicamento anticoagulante más utilizado en el mundo, mientras que el acenocumarol lo es en España. Los 


\section{Manejo postoperatorio de los anticoagulantes orales}

Vol. 3, núm. 2., (2019)

Ana Gabriela Garzón Medina; Wilson Moisés Ortiz Contreras; Karina Fernanda Veliz Guadamud; José María Cabezas Montes

efectos farmacológicos de los anticoagulantes orales son su efecto anticoagulante y su efecto antitrombótico.

En relación a su efecto anticoagulante aparece a las 12-24 horas de su administración mientras que el efecto antitrombótico aparece a los 3 días de tratamiento. El efecto antitrombótico aparece al alcanzar el estado de equilibrio entre la disminución en la síntesis de los factores vitamina $\mathrm{K}$ dependientes y la eliminación de los factores de coagulación en el plasma. La semivida de eliminación para los factores de la coagulación vitamina $\mathrm{K}$ dependientes son de 6, 24, 40 y 60 horas para los factores VII, IX, X, y II, respectivamente. Los anticoagulantes orales se utilizan en el tratamiento de la trombosis venosa, en la prevención de recurrencias del tromboembolismo venoso, en la prevención de pacientes con válvulas protésicas o con fibrilación auricular crónica, prevención en los procedimientos quirúrgicos con riesgo de trombosis moderado o elevado, o como tratamiento coadyuvante de la oclusión arterial coronaria donde los anticoagulantes orales tienen menos eficacia. El proceso de agregación es inhibido por varias sustancias naturales (generalmente prostaglandinas), que se producen en el endotelio y cuyo propósito es precisamente evitar que ocurra una coagulación intravascular sin previa lesión.

En cuanto a los nuevos anticoagulantes orales, se precisa que los mismos han demostrado ser más seguros que los AVK, principalmente en la reducción del riesgo de hemorragias intracraneales. No obstante, son fármacos que por su mecanismo de acción no están exentos de producir complicaciones hemorrágicas. Una de las principales limitaciones para el empleo de estos medicamentos ha sido la no disponibilidad de un antídoto específico que revierta de forma rápida 


\section{Manejo postoperatorio de los anticoagulantes orales}

Vol. 3, núm. 2., (2019)

Ana Gabriela Garzón Medina; Wilson Moisés Ortiz Contreras; Karina Fernanda Veliz

Guadamud; José María Cabezas Montes

su efecto anticoagulante en caso de hemorragia grave, mientras que los fármacos AVK cuentan con un antídoto específico, la vitamina $\mathrm{K}$.

Finalmente se puede entender que, los anticoagulantes de acción directa, donde se incluye rivaroxaban, dabigatran, apixaban y edoxaban, son medicamentos que actúan de manera diferente al acenocumarol y aldocumar, bloqueando el sistema de la coagulación en un determinado punto. Esto permite usar una dosis fija, una o dos veces al día, sin necesidad de realizar los controles de hemostasia (o coagulación) habituales realizados con acenocumarol, dado que las concentraciones del fármaco en sangre son más previsibles y estables. Pues, mediante su utilización los pacientes logran prevenir la formación de coágulos sanguíneos. También evitan que los ya existentes se hagan más grandes. Los coágulos en las arterias, venas y corazón pueden causar ataques, derrames cerebrales y bloqueos.

\section{Conclusiones}

Una vez que se valoraron los diferentes contenidos que estructuran el presente artículo, se puede considerar como argumentaciones generales las siguientes: Aunque los nuevos anticoagulantes orales representarán un avance en el tratamiento anticoagulante, se plantean retos y cuestiones que no están completamente clarificadas. En otras palabras, el manejo antes y después de la cirugía, procedimientos invasivos, anestesia neuroaxial, hemorragia, necesidad de cirugía urgente o reversión urgente sin disponibilidad de antídotos. Con los AVK, disponer de la determinación del INR para conocer el grado de efecto anticoagulante y qué estrategia de reversión es más adecuada a la situación clínica facilita el manejo clínico. 


\section{Manejo postoperatorio de los anticoagulantes orales}

Vol. 3, núm. 2., (2019)

Ana Gabriela Garzón Medina; Wilson Moisés Ortiz Contreras; Karina Fernanda Veliz Guadamud; José María Cabezas Montes

Cabe agregar que los nuevos anticoagulantes orales, no es necesaria la monitorización sistemática, en ciertas circunstancias puede ser recomendable. Por ejemplo, para evaluar el cumplimiento, en situación de complicación tromboembólica o hemorrágica, antes de procedimientos invasivos También puede ser recomendable conocer las concentraciones para ver si es posible realizar una trombolisis en caso de ictus o infarto.

Por otra parte, los anticoagulantes orales modernos como el dabigatrán, rivaroxabán y apixabán son sustratos de la glucoproteína P (GP-P), que es un transportador que funciona como una bomba de flujo que dificulta la absorción de determinadas sustancias. Los inhibidores de la GP-P aumentan la absorción, por lo que causan incremento en las concentraciones plasmáticas, e inductores de la GP-P las disminuyen. Estos inhibidores de la GP-P (como la amiodarona, ketoconazol, quinidina y verapamilo) incrementan la exposición del dabigatrán, y los inductores como la rifampicina la disminuyen. Es probable que otros inductores e inhibidores de la GP-P influyan en las concentraciones de los medicamentos citados.

En consecuencia, los anticoagulantes orales de nueva generación, dabigatran y rivaroxaban, son los primeros de una nueva serie de antitrombóticos no relacionados con los cumarínicos, que inhiben de forma selectiva y directa la trombina y el factor Xa, respectivamente. De modo general, los nuevos anticoagulantes orales comparten las mismas contraindicaciones relativas y precauciones que cualquier antitrombótico, las cuales se pueden resumir en aquellas situaciones donde el riesgo hemorrágico del paciente esté aumentado. Estarán contraindicados en pacientes con hemorragia activa o diátesis hemorrágica importante. Finalmente, como cualquier otro fármaco, si existe alergia, intolerancia o hipersensibilidad al principio activo o sus excipientes. 


\section{Manejo postoperatorio de los anticoagulantes orales}

Vol. 3, núm. 2., (2019)

Ana Gabriela Garzón Medina; Wilson Moisés Ortiz Contreras; Karina Fernanda Veliz

Guadamud; José María Cabezas Montes

En relación a los anticoagulantes orales son medicamentos que hacen que la sangre tarde más tiempo en coagular, con el fin de evitar la trombosis y/o embolia. El contenido en vitamina $\mathrm{K}$ de la dieta puede interferir en la eficacia del tratamiento, favoreciendo tanto un exceso como un defecto de esta. Existen diferentes formas de vitamina K, pero la más importante es la vitamina K1, llamada también filoquinona, es una vitamina liposoluble que se encuentra en las verduras de hojas verdes y juega un papel importante en la coagulación de la sangre.

\section{Bibliografía.}

Gómez, J. (2019). Métodos y Técnicas de Lectura. España: Paidós.

Márquez, B. (2016). Fisiología de la Coagulación. Es Salud, 17.

Mateo, S. (2017). Nuevos Anticoagulantes Orales. Las Condes, 18-25.

Naranjo, G. (2017). Anestesiología. Médica España, 15-21.

Ordovás, K. (2018). Anticoagulantes Naturales. Cardiología, 18-22.

Peña, R. (2017). Visión del Trabajo Monográfico. México: Limosa.

Ramos, P. (2017). Metodología y Paradigma. México: Gedisa.

$$
\text { (c) (1) () (2) }
$$

\section{RECONOCIMIENTO-NOCOMERCIAL-COMPARTIRIGUAL}

CC BY-NC-SA

ESTA LICENCIA PERMITE A OTROS ENTREMEZCLAR, AJUSTAR Y CONSTRUIR A PARTIR DE SU OBRA CON FINES NO COMERCIALES, SIEMPRE Y CUANDO LE RECONOZCAN LA AUTORÍA Y SUS NUEVAS CREACIONES ESTÉN BAJO UNA LICENCIA CON LOS MISMOS TÉRMINOS. 